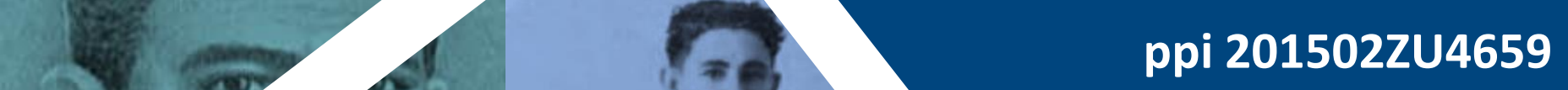

Esta publicación científica en formato digital es continuidad de la revista impresa

SSN 0254-0770/e-ISSN 2477-9377/Depósito legal pp $197802 Z U 38$

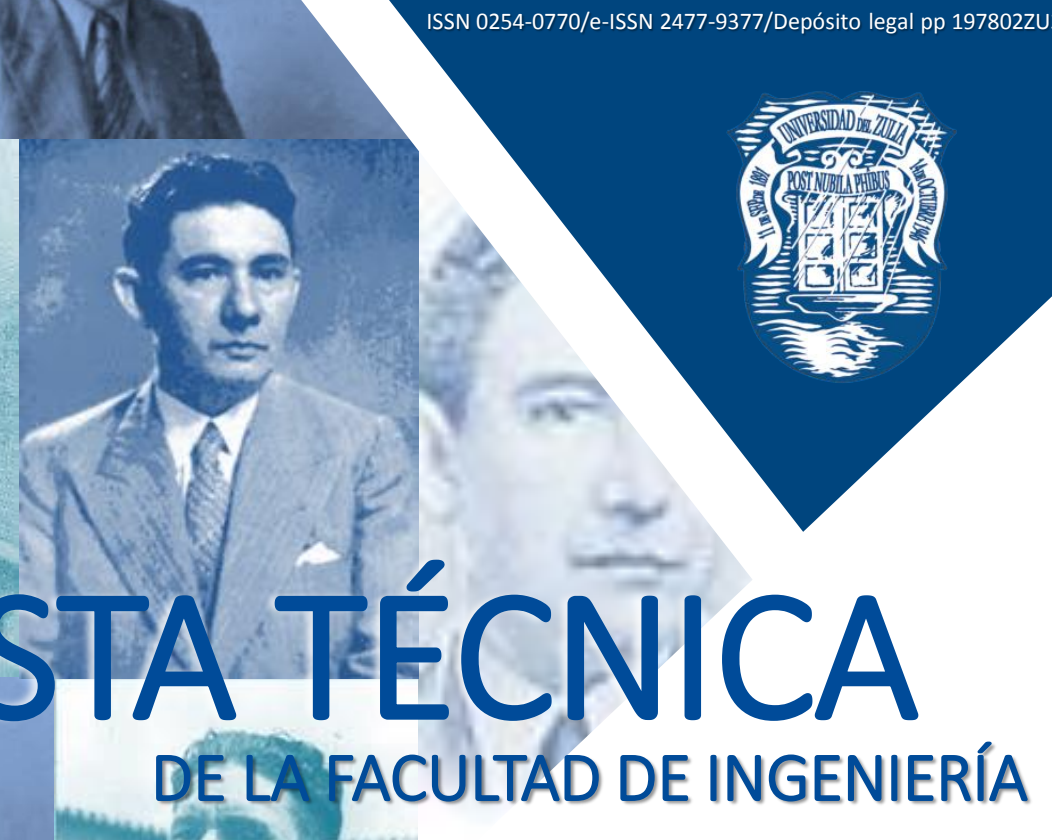
que está indizada en las publicaciones de referencia y comentarios:

- REDALYC

- REDIB

- SCIELO

- DRJI

- INDEX COPERNICUS INTERNATIONAL

- LATINDEX

- DOAJ

- REVENCYT

- CHEMICAL ABSTRACT

- MIAR

- aerospace dATABASE

- CIVIL ENGINEERING ABTRACTS

- METADEX

- COMMUNICATION ABSTRACTS

- ZENTRALBLATT MATH, ZBMATH

- ACTUALIDAD IBEROAMERICANA

- BIBLAT

- PERIODICA

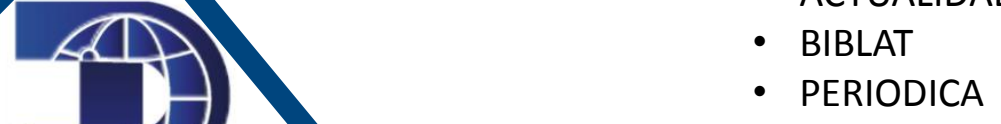




\title{
Efecto del Manejo Agroecológico sobre el Crecimiento de Plántulas de Theobroma cacao L. en Fase de Vivero
}

\author{
Rosa Isela Villasmil Villasmil* (id, Juan Carlos Nava Luzardo ${ }^{(}$, Elvis Portillo \\ Páez ${ }^{(D,}$ Eduar Díaz Barrios
}

Facultad de Agronomía, Universidad del Zulia. Apartado postal 4005. Maracaibo, Venezuela. *Autor de correspondencia: rosa.villasmil@gmail.com

https://doi.org/10.22209/rt.v45n1a02

Recepción: 07 de diciembre 2020 | Aceptación: 13 de septiembre de 2021 | Publicación: 28 de diciembre de 2021

\section{Resumen}

El cacao Theobroma cacao L. ha sido un rubro muy importante en la producción agrícola del país durante muchos años, contribuyendo con su desarrollo económico y social. El manejo agronómico actual a las plántulas de cacao en vivero, implica el uso de agroquímicos, que pudiesen ocasionar un perjuicio ambiental. En esta investigación se evaluó el efecto del manejo agroecológico sobre el crecimiento de plántulas de cacao en fase de vivero en el municipio Sucre del estado Zulia, Venezuela. El diseño experimental fue totalmente al azar con 5 tratamientos y 10 repeticiones: T1, fórmula completa 12-12-17; T2, lixiviado de raquis de plátano + Trichoderma harzianum + Beauveria bassiana; T3, vermicompost líquido + Trichoderma harzianum + Beauveria bassiana; T4, lixiviado de raquis de plátano + Trichoderma harzianum + trampa y T5, vermicompost líquido + Trichoderma harzianum + trampa. Los datos de crecimiento de las plántulas se analizaron siguiendo la metodología de medidas repetidas, observándose que en fase de vivero, las mismas se pueden manejar sin el uso de agroquímicos. El modelo de estimación para el tamaño de plántulas de cacao a través del tiempo, permitiría obtener información en un momento determinado de su proceso de crecimiento.

Palabras clave: Beauveria bassiana; lixiviado de raquis; Trichoderma harzianum; vermicompost.

\section{Effect of Agroecological Management on the Growth of Theobroma cacao L. Seedlings in the Nursery Phase}

\begin{abstract}
The Theobroma cacao L. cocoa has been an important item in the agricultural production of the country for many years, contributing with its economic and social development. The current agronomic management to cacao seedlings in nursery implies the use of agrochemicals that could cause environmental damage. In this research the effect of the agronomic management on the growth of cocoa seedlings in nursery phase in Sucre municipality, Zulia State, Venezuela was evaluated. The experimental design was totally randomized with 5 treatments and 10 repetitions: T1, complete formula 12-12-17; T2, plantain rachis leachate + Trichoderma harzianum + Beauveria bassiana; T3, liquid vermicompost + Trichoderma harzianum + Beauveria bassiana; 4 , plantain rachis leachate + Trichoderma harzianum + trap and T5, liquid vermicompost + Trichoderma harzianum + trap. The growth data of seedlings were analyzed following the repeated measures methodology, observing that in the nursery phase, seedlings can be managed without the use of agrochemicals. The estimation model for the size of cocoa seedlings over time would allow information to be obtained at a given moment in the growth process.
\end{abstract}

Key words: Beauveria bassiana; rachis leachate; Trichoderma harzianum; vermicompost. 


\title{
Efeito do Manejo Agroecológico no Crescimento de Mudas de Theobroma cacao L, na Fase do Viveiro
}

\begin{abstract}
Resumo
O cacau Theobroma cacao L. é há muitos anos um item muito importante na produção agrícola do país, contribuindo para o seu desenvolvimento econômico e social. $\mathrm{O}$ atual manejo agronômico das mudas de cacau em viveiro implica no uso de agroquímicos, que podem causar danos ambientais. Esta pesquisa avaliou o efeito do manejo agroecológico no crescimento de mudas de cacau em fase de viveiro no município de Sucre no estado de Zulia, Venezuela. $\mathrm{O}$ delineamento experimental foi inteiramente casualizado con 5 tratamientos y 10 repeticiones: T1, fórmula completa 12-12-17; T2, lixiviado de banana raquis + Trichoderma harzianum + Beauveria bassiana; T3, vermicomposto líquido + Trichoderma harzianum + Beauveria bassiana; T4, lixiviado de banana raquis + Trichoderma harzianum + armadilha e T5, vermicomposto líquido + Trichoderma harzianum + armadilha. Os dados de crescimento das mudas foram analisados seguindo a metodologia de medidas repetidas, observando-se que na fase de viveiro podem ser manejadas sem o uso de agroquímicos. O modelo de estimativa do tamanho das mudas de cacau y longo do tempo permitirá obtener informação num momento determinado do seu proceso de crescimento.
\end{abstract}

Palavras-chave: Beauveria bassiana; lixiviado de rachis; Trichoderma harzianum; vermicomposto.

\section{Introducción}

El cultivo de cacao (Theobroma cacao L.) es un rubro esencial para el sustento de 40-50 millones de personas en todo el mundo, ubicadas tanto en África de pequeños agricultores a y Asia, como en América Latina, cuyos productores, incluyendo más de 5 millones de pequeños agricultores que generan alrededor del $90 \%$ del cacao mundial (FAOSTAT, 2017). Este rubro, de gran importancia en lo social, alimentario, económico, entre otros, ha generado durante muchos años numerosas fuentes de empleos directos e indirectos, estando muy ligado a aspectos históricos y socioculturales, dada la importancia, no solo como fuente de ingreso a diferentes comunidades, sino además como aspecto notorio de su patrimonio e identidad, siendo un producto agrícola para la exportación en muchos países (García et al., 2017).

En la zona Sur del Lago de Maracaibo, estado Zulia, Venezuela, se cultiva tradicionalmente por su excelente aroma y calidad para la chocolatería, así como su atractivo precio en el mercado internacional (Moreno et al., 2020). No obstante el cacao se debería manejar con un criterio de sostenibilidad y sustentabilidad, garantizando su permanencia en el tiempo y con ello el arraigo de los productores en sus unidades de producción, siendo el municipio Sucre del estado Zulia una zona productora de cacao por excelencia, por presentar condiciones agroclimáticas idóneas para el desarrollo y producción del mencionado rubro (Fundacite-Zulia, 2010).

El manejo que actualmente se le da a las plantas de cacao en vivero, implica el uso de insumos agrícolas externos conocidos como agroquímicos, los cuales son utilizados para el control de enfermedades e insectos, lo cual implica un alto costo para el productor, además del impacto ambiental que pudiese estar ocasionando y el daño que representa para su salud (Palencia et al., 2014). El cacao en la etapa de vivero se ve afectado por el ataque de un gran número de insectos, como áfidos, barrenador del tallo, gusanos medidores o defoliadores hormigas, trips, ácaros y enfermedades como la mazorca negra, entre otras. El uso de técnicas agroecológicas permite mantener a los controladores biológicos de los insectos plaga sin causarles daño, con aplicaciones de repelentes orgánicos en el momento oportuno (Estrada et al., 2014).

Según Nava (2020) es imprescindible que se proporcione una mejor calidad de vida social y económica para que los productores de cacao puedan ser cada día más competitivos, incorporando experiencias en sus procesos y, cuidando el ambiente. De esta manera, el manejo agroecológico surge como una alternativa que sustituye el uso de los agroquímicos por productos naturales, sin riesgos de contaminación. Para Cevallos et al. (2019) los productores deberían lograr el aumento de la productividad sin ocasionar un impacto negativo en los recursos naturales. Por lo tanto, se deberían tomar acciones sobre las prácticas realizadas y productos aplicados, y planificar las próximas actividades a realizar. Con la opción de diferentes alternativas ecológicas, se pueden establecer un programa de control adecuado para disminuir el grave deterioro ambiental y social causado por la aplicación continua e 
indiscriminada de agroquímicos (Nava et al., 2021). En este sentido, hay que considerar los diferentes escenarios de producción, resaltando las bondades en calidad, menor contaminación, beneficios para la salud, entre otros, en mejora de la calidad de vida de la población (Cedeño et al., 2021).

De acuerdo con el contexto anterior, para el manejo agroecológico, se cuenta con: i) lixiviado de raquis plátano (L), que es producido por la descomposición del raquis de plátano y, es usado como abono orgánico líquido, por ser rico en micro elementos y en potasio, al mismo tiempo que contiene gran cantidad de microorganismos, con propiedades plaguicidas; ii) Beauveria bassiana (B), que un hongo que crece de forma natural en los suelos, su poder entomopatógeno es capaz de parasitar a insectos de diferentes especies; iii) Trichoderma harziarum (T), que es un hongo beneficioso para las plantas, utilizado como controlador biológico contra diversos patógenos vegetales y que puede ser aplicado para el control de un grupo importante de patógenos del suelo; y iv) vermicompost (V), que es un abono orgánico, producto de la descomposición de la materia orgánica, realizada únicamente por especies de lombrices, específicamente del género Eisenia, siendo la más utilizada la lombriz roja californiana (Eisenia foetida), la cual realiza un proceso de descomposición más rápidamente, transformando la materia orgánica en lo que se denomina humus de lombriz o V (Estrada et al., 2014). Por su parte, la fórmula completa NPK 12-12-17, es un fertilizante sólido granulado; utilizado en muchos cultivos, entre ellos, el cacao.

Considerando entonces la importancia de conocer diferentes alternativas agroecológicas para el cultivo del cacao y otros rubros, con la finalidad de minimizar el impacto negativo en los seres humanos, animales y el ambiente, en pro de mantener un equilibrio ecológico sustentable; el objetivo de la presente investigación fue, evaluar el efecto del manejo agroecológico sobre el crecimiento de plántulas de Theobroma cacao L. en fase de vivero, mediante la aplicación de distintas combinaciones de L. B, T y V.

\section{Materiales y Métodos}

\section{Condiciones experimentales}

La investigación se realizó en el municipio Sucre, en la zona Sur del Lago de Maracaibo, en la siguiente ubicación geográfica: $09^{\circ} 22^{\prime \prime}$ latitud N 7054" longitud W, limitando al norte con el Lago de Maracaibo, al sur con la cordillera Andina, al este con el estado Trujillo y al oeste con el municipio Francisco Javier Pulgar; La zona cuenta con una superficie de $1.174 \mathrm{~km}^{2}$, una temperatura media de $32{ }^{\circ} \mathrm{C}$, altura de aproximadamente $6 \mathrm{msnm}$ y suelos de tipo franco arenoso. La humedad relativa máxima anual es de 96,58 \% y la mínima es de 52,06 \%. La velocidad del viento máxima anual es de $36,72 \mathrm{~km} / \mathrm{h}$ y la mínima de $17,61 \mathrm{~km} / \mathrm{h}$; el pH del suelo es de 5,2 - 6,5; con un contenido bajo de materia orgánica (Corpozulia, 2018).

\section{Diseño de la investigación}

El diseño experimental fue totalmente al azar con 5 tratamientos y 10 repeticiones para un total de cincuenta observaciones. La unidad experimental fue una plántula de cacao sembrada en una bolsa de $2 \mathrm{~kg}$. Se realizaron aplicaciones de los tratamientos, observaciones y mediciones cada $15 \mathrm{~d}$, por $135 \mathrm{~d}$.

\section{Tratamientos}

En la Tabla 1 se muestran los 5 tratamientos utilizados: T1, fórmula completa NPK 12-12-17; T2, L + T + B; T3, V + T + B; T4, L + T + trampa y T5, V + T + trampa.

\section{Variables evaluadas}

Las variables evaluadas durante los ensayos, fueron las siguientes: i) brotación de las plantas: se realizó el conteo de plantas brotadas en cada unidad experimental por tratamiento; ii) altura de las plantas (A) (longitud): semanalmente se midió con una cinta métrica, desde la base de la plántula hasta la hoja más alta, expresándose en centímetros; iii) diámetro (D): semanalmente se midió con una cinta métrica el diámetro del tallo, expresándose en centímetros; y iv) número de hojas (N): semanalmente se contó la cantidad de hojas funcionales presentes en las plántulas. 
Tabla 1. Tratamientos en el manejo agroecológico sobre el crecimiento de plántulas de cacao en fase de vivero en el municipio Sucre del estado Zulia.

\begin{tabular}{cl}
\hline Tratamiento & \multicolumn{1}{c}{ Característica } \\
\hline 1 & Fertilizante, fórmula completa NPK 12-12-17,2 g/p. \\
2 & $\begin{array}{l}\text { Lixiviado de raquis de plátano } 90 \%+\text { agua } 10 \%)+ \text { aplicación de } \\
\text { Trichoderma harzianum + Beauveria bassiana. }\end{array}$ \\
3 & $\begin{array}{l}\text { Vermicompost líquido, 10\%+90\% de agua + aplicación de Trichoderma } \\
\text { harzianum + Beauveria bassiana. }\end{array}$ \\
4 & $\begin{array}{l}\text { Lixiviado de raquis de plátano, } 60 \%+40 \% \text { de agua + aplicación de } \\
\text { Trichoderma harzianum + trampa. }\end{array}$ \\
& $\begin{array}{l}\text { Vermicompost líquido, } 5 \%+95 \% \text { agua }+ \text { aplicación de Trichoderma } \\
\text { harzianum + trampa. }\end{array}$ \\
\hline
\end{tabular}

\section{Preparación del sustrato}

Como sustrato se utilizó tierra negra, con alto contenido de materia orgánica, la cual fue cernida (tamaño de poro de alrededor de $1,5 \mathrm{~mm}$ ) en un tamiz o cedazo, eliminando así partículas grandes tales como piedras y otros cuerpos extraños.

\section{Selección y preparación de las semillas}

Se seleccionaron mazorcas de cacao híbrido Sur del Lago en un estado de madurez óptima, libre de insectos y enfermedades. Una vez seleccionadas las mazorcas, se procedió a abrirlas cuidadosamente, con un cuchillo, seguidamente se extrajeron las semillas de forma manual desprendiéndolas de la placenta, se seleccionaron las semillas que estaban en la parte media de la mazorca, luego se le retiró el mucílago, que es la pulpa de color blanco y dulce que las recubre, para facilitar la germinación. Las semillas sin mucilago se lavaron con agua limpia, para eliminar los restos del material utilizado durante el proceso de eliminación de la baba o pulpa; posteriormente se colocaron en la sombra durante 3 días para facilitar la germinación.

\section{Llenado de bolsas y siembra de semillas}

Para sembrar las semillas de cacao se utilizaron bolsas negras de polietileno, perforadas, con una capacidad de $2 \mathrm{~kg}$. Una vez seleccionadas las bolsas, se procedió a llenarlas totalmente con el sustrato, compactándolo al hacer presión con los dedos o golpear con suavidad la base de la bolsa llena contra el suelo. Una vez preparadas las bolsas, se regaron para humedecer el sustrato, y se sembraron las semillas haciendo un orificio en el centro de la bolsa; se dispuso una semilla ya germinada por bolsa, con la radícula o brote, (primera raíz que emerge), hacia abajo y se enterró un tercio, o mitad de la semilla. Luego, las bolsas, se acomodaron en una superficie uniforme del vivero, en filas de 10 unidades, con una separación entre filas de $10 \mathrm{~cm}$, para facilitar las labores de mantenimiento y que las plantas se desarrollaran uniformemente.

\section{Manejo en el vivero}

Durante esta fase se realizaron las labores de mantenimiento necesarias para garantizar un buen desarrollo de las plántulas de cacao, tales como: i) control de malezas: se realizó el control de malezas de forma manual cada 15 días, para evitar la competencia con las plántulas de cacao por nutrientes, espacio, luz, agua, entre otros; ii) control de insectos y enfermedades: se realizó el control de insectos con la colocación de trampas, las cuales fueron hechas con foami de color fosforescente impregnadas de aceite vegetal, para atraer a los insectos que pudieran atacar a las plántulas en su primera etapa de desarrollo. Estas se colocaron una por cada dos hileras de plantas y se aplicó B como bioinsecticida, y para evitar la aparición de hongos causantes de enfermedades fúngicas se aplicó T; iii) riego: el agua para riego se aplicó mediante un sistema por aspersión, con una frecuencia de dos veces al día; y iv) fertilización: se realizaron aplicaciones de fertilizantes orgánicos tales como $\mathrm{V}$ o L y una fórmula completa de fertilizante comercial NPK 12-12-17 (agroquímico), según fuere el tratamiento. 


\section{Análisis estadístico}

Los datos de crecimiento de la plántulas se analizaron siguiendo la metodología de medidas repetidas, ajustando la estructura de covarianzas con un modelo autoregresivo de primer orden, mientras que para estimar el efecto fijo de los tratamientos a través del tiempo, se ajustaron distintos modelos, seleccionándose el modelo polinómico de tercer orden con la Ecuación 1:

$$
\mathrm{Y}=\alpha+\beta \mathrm{X}+\gamma \mathrm{X} 2+\delta \mathrm{X} 3
$$

Donde: Y representa la variable respuesta; $\alpha, \beta, \gamma, \mathrm{y} \delta$ los parámetros a estimar y X el tiempo en d.

Se realizó un análisis de la varianza (ANOVA) sobre las variables en estudio para demostrar el efecto de la aplicación de los diferentes tratamientos, utilizando la prueba de comparación de medias de Tukey y el procedimiento Proc Mixed del paquete estadístico SAS® (SAS, 2020). El programa Microsoft Office Excel 2010 se usó para elaborar la base de datos y los gráficos de variabilidad.

\section{Resultados y Discusión}

\section{Altura de las plántulas}

Los resultados obtenidos no mostraron diferencias significativas entre los tratamientos $(\mathrm{P}>0,05)$, al ser analizada A. En la Figura 1 se observa que pese a que los valores de altura alcanzado por las plántulas de cacao de T5 obtuvieron una mayor altura con $28,39 \mathrm{~cm}$; T4, T2 y T3 presentaron alturas de planta muy cercanos, con: 26,32; 25,80 y 24,95 cm, respectivamente, seguidas de las plántulas del T1, las cuales alcanzaron una menor altura de 15,50 $\mathrm{cm}$ a los $135 \mathrm{~d}$. Los valores de altura alcanzados por las plántulas de T2, T3, T4 y T5 reflejan que se puede realizar un manejo con estos productos con excelente respuesta.

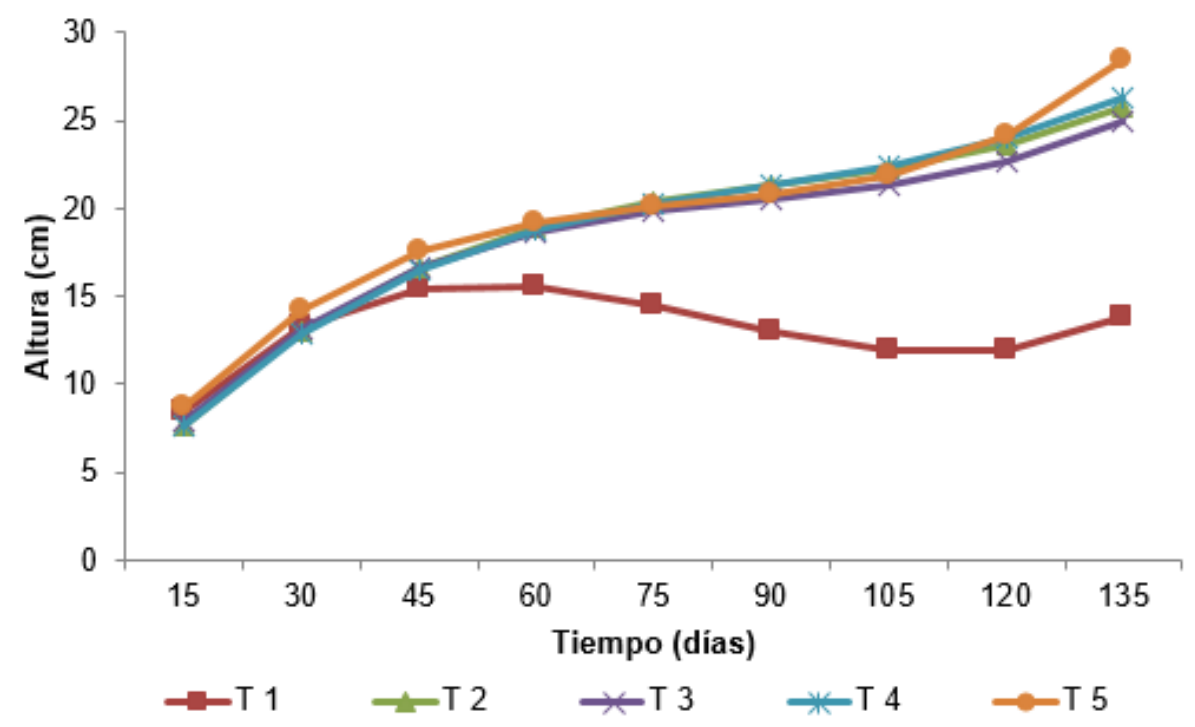

Figura 1. Altura de las plántulas de cacao en función del tiempo (días) en fase de vivero en el municipio Sucre. La codificación de los tratamientos se define en la Tabla 1.

Estos resultados coinciden con los reportados por Dorado (2019), con valores de 21 a $28 \mathrm{~cm}$ a los $120 \mathrm{~d}$ sobre el crecimiento y desarrollo de plántulas de cacao en vivero, y por Ramírez et al. (2018), quienes utilizaron abonos orgánicos, igual en fase de vivero. También son comparables con los reflejados por Ormeño (2011), con valores de alrededor de $25 \mathrm{~cm}$; señalando que la adecuada utilización de abonos orgánicos favorecerá a las plántulas 
de cacao al momento del trasplanté, brindándoles mejores oportunidades para su supervivencia. Así, mediante el manejo agroecológico se pueden obtener plántulas con alturas adecuadas paras ser llevadas a campo, con un sentido ecológico y de sostenibilidad, conociendo diferentes alternativas naturales, antes de utilizar agroquímicos.

\section{Modelos para la estimación de la altura de plántulas}

El modelo que mejor se ajustó a los datos observados para la altura de las plántulas de cacao, fue el polinomio de tercer grado y sus estimaciones para cada tratamiento pueden ser observadas en la Tabla 2.

Tabla 2. Modelos para la estimación de la altura de plántulas de cacao en función del tiempo (días) en fase de vivero en el municipio Sucre. La codificación de los tratamientos se define en la Tabla 1.

\begin{tabular}{cc}
\hline Tratamiento & Modelo \\
\hline 1 & $0,1455+0,6874 *$ Días $-0,00947 *$ Días $2+0,000038 *$ Días3 \\
2 & $0,1455+0,5809 *$ Días $-0,00573 *$ Días $2+0,000021 *$ Días3 \\
3 & $0,1455+0,6036 *$ Días $-0,00635 *$ Días $2+0,000024 *$ Días3 \\
4 & $0,1455+0,5753 *$ Días $-0,00566 *$ Días $2+0,000021 *$ Días3 \\
5 & $0,1455+0,6804 *$ Días $-0,00808 *$ Días $2+0,000034 *$ Días3 \\
\hline
\end{tabular}

El modelo para T1 corresponde a las menores alturas de planta, entre 11,66 y 14,56 cm; al compararlas con los otros tratamientos, los cuales mostraron un comportamiento muy similar. Este modelo de estimación permitiría a los productores, estudiantes, técnicos, investigadores, entre otros, obtener información sobre el comportamiento de las plántulas en un momento determinado de su proceso de crecimiento durante la fase de vivero; de esto depende en mayor grado la producción de plántulas sanas, vigorosas y con una mayor uniformidad. Se incluiría en el modelo de estimación el número correspondiente a los días que las plántulas tengan, según el tratamiento, para poder conocer las condiciones en las que se encuentran durante su permanencia en el vivero.

Ormeño et al. (2018), por su parte, indicaron la importancia de realizar un manejo agroecológico, y que el crecimiento de las plántulas depende de la calidad del sustrato utilizado y de los abonos orgánicos, por lo que se deben utilizar diferentes opciones como alternativa para el manejo sustentable. En este contexto, se requiere de material vegetal proveniente de viveros, donde se realice un manejo amigable con el ambiente Osorio et al. (2018). También Suárez et al. (2021) señalaron que se debe realizar un manejo agroecológico con posibilidades de obtener el rendimiento potencial, de acuerdo con las características de los agroecosistemas. De esta manera, se debería realizar una orientación hacia la optimización del uso de los recursos y la protección ambiental, pretendiendo satisfacer las necesidades actuales sin comprometer el ambiente. Es necesario que se siga trabajando en conocer y utilizar las diferentes alternativas naturales que existen, antes de aplicar un determinado tipo de agroquímico.

\section{Brotación}

En cuanto al porcentaje de brotación de todos los tratamientos evaluados, no se registraron diferencias significativas $(\mathrm{P}>0,05)$, al ser analizados estadísticamente, ya que todas las semillas de cacao realizaron la brotación. Estos resultados coinciden con los reportados por Ramírez et al. (2018), con 100 \% de brotación de la semilla de cacao, en condiciones de vivero a los $15 \mathrm{~d}$.

\section{Diámetro del tallo}

La variable D no arrojó diferencias significativas entre los tratamientos $(\mathrm{P}>0,05)$. En la Figura 2 pueden observarse sus valores para los diferentes tratamientos. A nivel de las pruebas de Tukey se observaron diferencias mínimas, donde las plántulas de T2, T3, T4 y T5 presentaron 8,07; 7,44; 7,22 y 8,16 cm, respectivamente, durante los 135 d, mientras que las plantas de T1 arrojaron el menor diámetro con 4,59 cm. Ramírez et al. (2018) y Dorado (2019) presentaron resultados de diámetro del tallo de alrededor de $8 \mathrm{~cm}$, coincidiendo con los resultados obtenidos en esta investigación. 


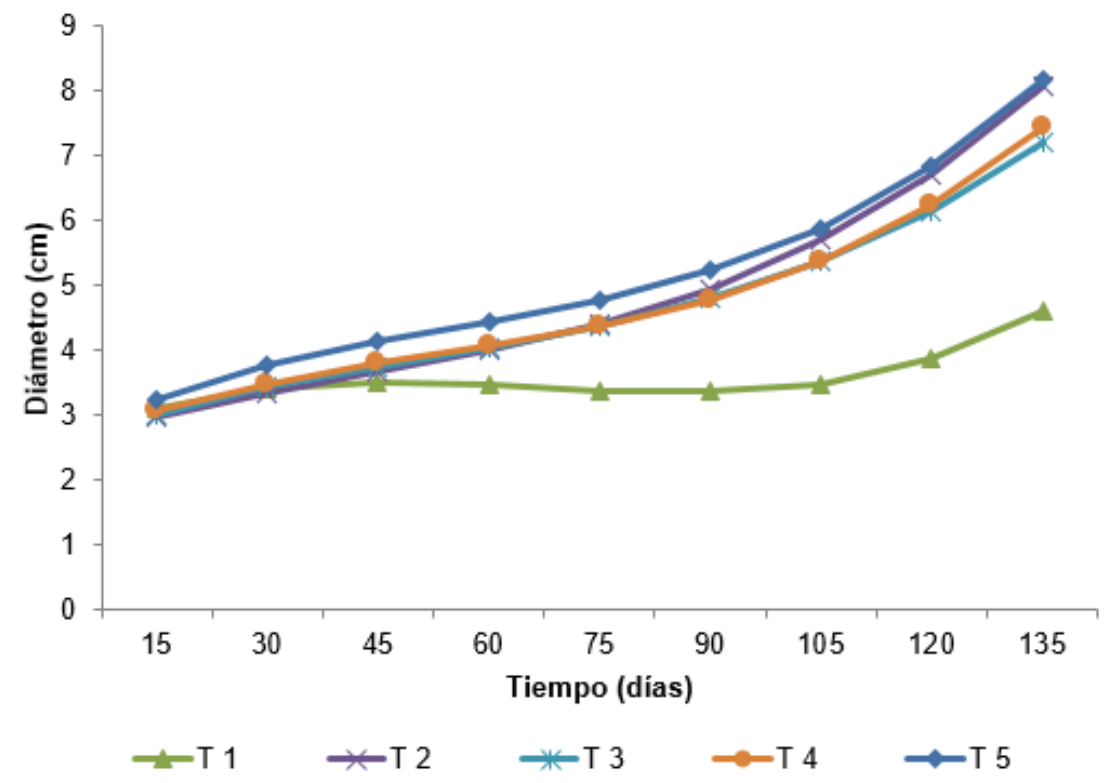

Figura 2. Diámetro del tallo en función del tiempo (días) de las plántulas de cacao en fase de vivero en el municipio Sucre. La codificación de los tratamientos se define en la Tabla 1.

\section{Número de hojas}

El análisis estadístico tampoco mostró variaciones significativas $(\mathrm{P}>0,05)$ entre los tratamientos para la variable N; se observó que las plántulas de T5 alcanzaron el mayor número de hojas con 20, seguidas por las del T2, T4 y T3 con 20, 20 y 19, respectivamente, siendo las plantas del T1 las que presentaron el menor número de hojas con 8 (Figura 3). Reflejándose que todos los productos de origen biológico estudiados representan una alternativa en el manejo agroecológico de las plántulas de cacao.

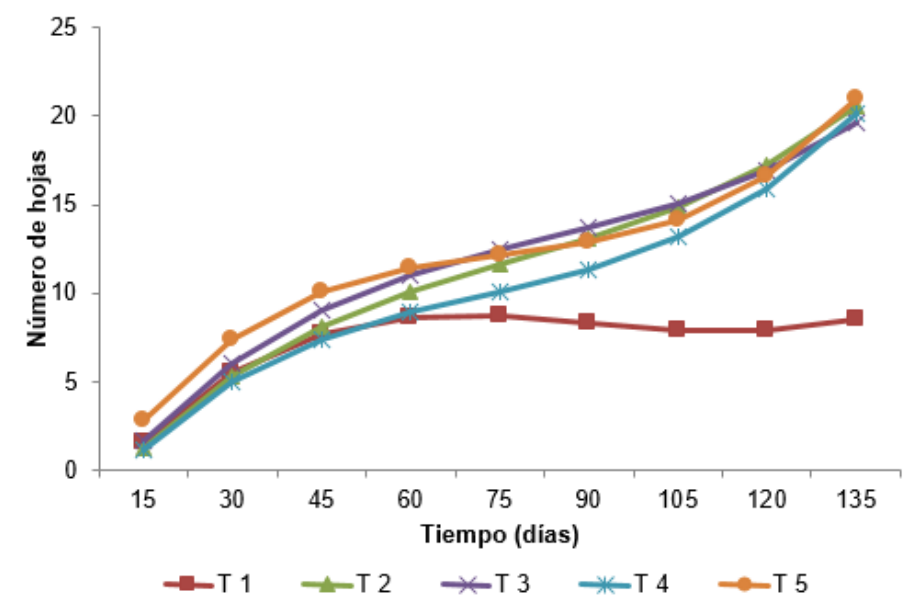

Figura 3. Número de hojas en función del tiempo (días) de las plántulas de cacao en fase de vivero en el municipio Sucre. La codificación de los tratamientos se define en la Tabla 1. 


\section{Manejo agroecológico}

En fase de vivero, las plántulas de cacao, se pueden manejar sin el uso de agroquímicos. Todos los tratamientos con los productos de origen biológico estudiados, representan una alternativa ecológica para el manejo de dichas plántulas en fase de vivero. Todas las plantas de T1 presentaron marchitamiento en las hojas, producto del hongo Phytophthora palmivora, a partir de los 105 días en la fase de vivero. Las plantas de T2, T3, T4 y T5 exhibieron la enfermedad pero fue controlada mediante las aplicaciones de $\mathrm{T}$, demostrando que las plántulas de cacao se pueden manejar en fase de vivero sin el uso de agroquímicos, siendo importante destacar que la producción orgánica es una alternativa que podría generar beneficios, ya que estos pueden obtenerse de materiales generados del proceso productivo dentro de las unidades de producción, lo que pudiera convertirse en una gran ventaja para todas las personas involucradas, logrando reducir el impacto ambiental que genera la utilización indiscriminada de agroquímicos durante la producción agrícola. Al respecto, Ludovic et al. (2013) señalaron que un alto número de productores manifiestan no tener conocimiento sobre el significado de producto orgánico, indicando que para lograr cosechas sostenibles, deben existir conocimientos técnicos para considerar los restos o desechos generados de su actividad. Según Nava et al. (2017) es necesario que se observe el entorno y el daño que se podría estar produciendo o se produciría en un futuro al ambiente, siendo necesario que se cuiden y se conserven los recursos naturales.

Durante el transcurso de esta investigación (135 d) todos los tratamientos presentaron hormigas y pulgones, los cuales fueron controlados con la aplicación del controlador biológico B, mientras que en las trampas solo se observaron entre tres y cuatro moscas, lo cual no representó un daño para las plántulas. Así, con la implementación de prácticas orgánicas, se podrían controlar los insectos del cacao en el vivero, evitando el uso de agroquímicos, tratando de utilizar insecticidas biológicos para su control (Gómez y Ormeño, 2013). También es importante señalar que se podría establecer un programa de manejo con la utilización de productos biológicos para mantener las plántulas de cacao sanas durante la fase de vivero. Al respecto, se podrían ejecutar prácticas de conservación de suelos, utilización de residuos de cultivos, abono de origen animal, abonos de desechos orgánicos, ente otros, siendo necesario un enfoque hacia la estabilidad de los sistemas y preservación del acceso a un ambiente saludable (Rincón et al., 2014).

Finalmente, se debe destacar que la producción orgánica es un sistema mediante el cual se busca mejorar las condiciones del ambiente y lograr mantener en el tiempo, desde el punto de vista social, económico y ecológico, un agroecosistema más saludable, implementando un programa de manejo a base de productos orgánicos. Se hace importante el manejo agroecológico del rubro desde la etapa de vivero, llevando a cabo la producción y mantenimiento de plantas de calidad, libres de enfermedades, con una buena conformación, y la ejecución de prácticas que logren garantizar la producción de plantas sanas, disminuyendo la utilización de agroquímicos, para así tratar de garantizar la protección al ser humano y al ambiente. De esta manera Füssel y Dinorah (2013), plantean la producción y uso de los abonos orgánicos como una alternativa económica para los productores, sin embargo, se debe estandarizar la producción para que la calidad de los mismos se mantenga en el tiempo. Por lo tanto, es importante implementar un sistema de manejo con la utilización de productos biológicos, tratando de disminuir el uso de agroquímicos, poner en práctica una serie de medidas ambientales que involucren tanto la protección a los seres humanos, como al ambiente.

\section{Conclusiones}

Todos los productos de origen biológico estudiados representan una alternativa ecológica para el manejo de las plántulas de cacao; pudiéndose utilizar con excelente respuesta en cuanto a brotación, tamaño de plántulas, diámetro del tallo y número de hojas. Se demostró que es factible realizar un manejo agroecológico sobre el crecimiento de plántulas de cacao en fase de vivero, sin el uso de agroquímicos; destacándose además que la producción orgánica es una alternativa viable para este importante rubro agrícola. Para ello, se puede elaborar un programa de fertilización con la utilización de productos orgánicos, estableciendo un manejo integrado de insectos y enfermedades, durante la fase de crecimiento de estas plántulas.

El modelo de estimación obtenido para el tamaño de plántulas de cacao a través del tiempo permitiría a los productores, estudiantes, técnicos, investigadores, entre otros, obtener información sobre el comportamiento de las plántulas en un momento determinado de su proceso de crecimiento durante la fase de vivero. 


\section{Referencias Bibliográficas}

Cedeño, J., Díaz, E., Conde, E., Cervantes, A., Avellán, L., Zambrano, M., Tobar, J., Estévez, S., Sánchez, A. (2021). Evaluación de la severidad de Sigatoka negra (Mycosphaerella fijiensis Morelet) en plátano "Barraganete" bajo fertilización con magnesio. Revista Técnica de la Facultad de Ingeniería de la Universidad del Zulia, 44(1), 411 .

Cevallos, M., Urdaneta, F., Jaimes, E. (2019). Desarrollo de sistemas de producción agroecológica: Dimensiones e indicadores para su estudio. Revista de Ciencias Sociales de la Facultad de Economía de la Universidad del Zulia, 15(3), 172-185.

Corpozulia. (2018). Caracterización de las condiciones climáticas y edáficas del municipio Colón. Dirección de información geográfica y estadística de Venezuela. Maracaibo: Corporación de Desarrollo de la Región Zuliana.

Dorado, E. (2019). Evaluación de complejos micorrízicos asociados al cultivo de plántulas de cacao (Theobroma cacao). Los Ríos: Universidad Técnica de Babahoyo.

Estrada, W., Romero, X., Moreno, J. (2014). Guía técnica del cultivo de cacao manejado con técnicas agroecológicas. San Salvador: CATIE.

FAOSTAT. (2017). Estadísticas de producción del cultivo del cacao [en línea] disponible en: http://faostat.fao.org/faostat/form/collection/Production.Crops [consulta: 11 diciembre 2020].

Füssel, J., Dinorah, S. (2013). El cacao, manejo en vivero y trasplante. Nindirí: Eco-tierra.

Fundacite-Zulia. (2010). La ruta del chocolate. Maracaibo: Ministerio del Poder Popular para la Ciencia, Tecnología e Industrias Intermedias.

García, G., Villar, L., Pérez, R. (2017). Tendencias de la investigación del cacao: oportunidades para la investigación en Santo Domingo de los Tsáchilas. SATHIRI, 12(2), 24-45.

Gómez, A., Ormeño, M. (2013). Selección de semilla y establecimiento de vivero para cacao. Maracay: Instituto Nacional de Investigaciones Agrícolas.

Ludovic, E., Rincón, N., Huerta, L., Rincón, R. (2005). Caracterización bajo un enfoque sostenible de las unidades de producción agrícola rurbanas en el municipio Maracaibo del estado Zulia. Revista de la Facultad de Agronomía, 22, 283-299.

Moreno, G., Sánchez, Z., Portillo, E., Ramírez, M. Gómez, A. (2020). Propagación asexual del Cacao Porcelana (Theobroma cacao L.) mediante la técnica de acodo aéreo. Revista de la Facultad de Agronomía de la Universidad del Zulia, 37(1), 31-37.

Nava, J. (2020). El maravilloso mundo del cultivo del cacao. Madrid: Editorial Académica Española.

Nava, J., Sánchez, A., Ortega, J. (2017). Gestión de planificación económica en el cultivo del plátano en el estado Zulia, Venezuela. Revista de la Facultad de Agronomía, 34, 371-396.

Nava, J., Urdaneta, T., Centanaro, P. (2021). Alternativas ecológicas de control de Mycosphaerella fijiensis en el cultivo de plátano. Revista Técnica de la Facultad de Ingeniería de la Universidad del Zulia, 44(3), 199-207.

Ormeño, M. (2011). Evaluación de diferentes abonos orgánicos en el crecimiento y desarrollo de plantas de cacao (Theobroma cacao). Instituto Nacional de Investigaciones Agrícolas, Mérida. Sociedad Interamericana de Horticultura Tropical, 54, 103-105.

Ormeño, M., Ovalle, A., Rey, J. (2018). Evaluación del efecto de abonos orgánicos líquidos en el crecimiento de plántulas de café (Coffea arabica L.) Revista de la Facultad de Agronomía, 35, 387-407. 
Osorio, M., Leiva, E.., Ramírez, R. (2017). Crecimiento de plántulas de cacao (Theobroma cacao L.) en diferentes tamaños de contenedor. Revista de Ciencias Agrícolas, 34(2), 73-82.

Palencia, G., Gómez, R., Gûiza, O. (2014). Nuevas tecnologías para instalar viveros y producir clones de cacao (Theobroma cacao L.). Bogotá: Corpoica.

Ramírez, M., Rodríguez, Y., Carrera, K. Asanza, M., Soria, S. (2018). Efecto de abonos orgánicos en el cultivo de Theobroma cacao L. en vivero del recinto El Capricho, provincia de Napo, Ecuador. Puyo: Universidad Estatal Amazónica.

Rincón, M., Morales, H., Nava, J., Gil, M. (2014). Desarrollo sostenible de la comunidad de Cherepta de la Sierra de Perijá, estado Zulia. Revista de la Facultad de Agronomía, 32, 381-406.

Statistical Analysis System (SAS®). (2020). SAS Studio user's guide: statistics. Version 15.1. Cary: SAS Institute Inc.

Suárez, G., Avendaño, C., Hernández, M., Rodríguez, L., Estrada, P., Salas, M. (2021). Zonificación edafoclimática del cultivo de cacao en el estado Chiapas. Revista Mexicana de Ciencias Agrícolas, 12(4), 629- 641. 


\section{REVISTA TECNICA}

DE LA FACULTAD DE INGENIERIA

UNIVERSIDAD DEL ZULIA

Vol. 45. №1, Enero - Abril, 2022

Esta revista fue editada en formato digital y publicada en Diciembre 2021, por el Fondo Editorial Serbiluz, Universidad del Zulia. Maracaibo-Venezuela

www.luz.edu.ve

www.serbi.luz.edu.ve

www.produccioncientificaluz.org 